\title{
Identification of therapeutic targets for breast cancer using biological informatics methods
}

\author{
XUEJIAN LIU ${ }^{1 *}$, YONGZHEN MA $^{2 *}$, WENCHUAN YANG ${ }^{1}$, XIA WU $^{1}$, LIHUA JIANG $^{1}$ and XIANGLI CHEN ${ }^{1}$ \\ ${ }^{1}$ Department of Oncology, The People's Hospital of Linyi Economic and Technological Development Zone, \\ Linyi, Shandong 276023; ${ }^{2}$ Department of Histological Embryology, Shandong Medical College, \\ Linyi, Shandong 276000, P.R. China
}

Received February 28, 2014; Accepted November 25, 2014

DOI: $10.3892 / \mathrm{mmr} .2015 .3565$

\begin{abstract}
The present study aimed to investigate the modular mechanisms underlying breast cancer and identify potential targets for breast cancer treatment. The differentially expressed genes (DEGs) between breast cancer and normal cells were assessed using microarray data obtained from the Gene Expression Omnibus database. Gene ontology (GO) and pathway enrichment analyses were performed in order to investigate the functions of these DEGs. Subsequently, the protein-protein interaction (PPI) network was constructed using the Cytoscape software. The identified subnetworks were further analyzed using the Molecular Complex Detection plugin. In total, 571 genes (241 upregulated and 330 downregulated genes) were found to be differentially expressed between breast cancer and normal cells. The GO terms significantly enriched by DEGs included cell adhesion, immune response and extracellular region, while the most significant pathways included focal adhesion and complement and coagulation cascade pathways. The PPI network was established with 273 nodes and 718 edges, while fibronectin 1 (FN1, degrees score, 39), interleukin 6 (IL6; degree score, 96) and c-Fos protein (degree score, 32 ) were identified as the hub proteins in subnetwork 2. These dysregulated genes were found to be involved in the development of breast cancer. The FN1, IL6 and FOS genes may therefore be potential targets in the treatment of breast cancer.
\end{abstract}

Correspondence to: Dr Xuejian Liu, Department of Oncology, The People's Hospital of Linyi Economic and Technological Development Zone, 117 Huaxia Road, Linyi, Shandong 276000, P.R. China

E-mail: xuejjll@hotmail.com

Dr Yongzhen Ma, Department of Histological Embryology, Shandong Medical College, 1 Jucai Sixth Road, Linyi, Shandong 276000, P.R. China

E-mail: yongzhzh@yeah.net

*Contributed equally

Key words: breast cancer, differentially expressed genes, protein-protein interaction network, therapeutic targets

\section{Introduction}

Breast cancer is a common type of tumor, which develops in the breast tissue. The majority of breast cancer cases occur in females, which may result from gender-associated characteristics (1) and certain hormones (2). Additional risk factors contributing to breast cancer development include genetics (3), obesity (4) and environmental pollution (5). Breast cancer is one of the leading causes of cancer-associated death amongst females, accounting for $\sim 23 \%$ of all cancer cases diagnosed in females (6). In 2008, 458,503 deaths resulted from breast cancer worldwide (7). Although the survival rate $(\sim 85 \%)$ for breast cancer is higher in Western countries, it is significantly lower in developing countries (6). Therefore, breast cancer is a global health concern.

Significant progress has been achieved in the elucidation of the pathological mechanisms underlying the development of breast cancer $(8,9)$. Certain genes have been identified to be involved in the progression of breast cancer. Breast cancer-specific gene 1 (BCSG1), also known as synuclein $\gamma$, was demonstrated to be overexpressed in breast tumor tissues and stimulated the metastasis of breast tumor cells (10). The elevated expression levels of nuclear receptor coactivator 3 (ncoa3; AIB1) and human epidermal growth factor receptor 2 (HER2) in breast cancer cells were demonstrated to contribute to tamoxifen resistance (11). Furthermore, certain significant pathways have also been found to play a crucial role in breast cancer. The HER2 tyrosine kinase pathway promoted hormone-independent growth and enhanced endocrine resistance in breast cancers (12). In addition, the activity of the Hedgehog signaling pathway in breast cancer cells was found to result in abnormal growth of the mammary duct and may therefore represent a candidate target for breast cancer treatment (13). Progress has been achieved in the elucidation of the mechanisms underlying breast cancer development, contributing towards the development of novel therapeutic methods. However, the present knowledge is insufficient.

In the present study, a biological informatics approach was used to analyze the gene expression profiles in breast cancer cells, while a functional analysis was performed in order to identify differentially expressed genes (DEGs) between breast tumor cells and matched normal tissues. Additionally, a protein-protein interaction (PPI) network was 

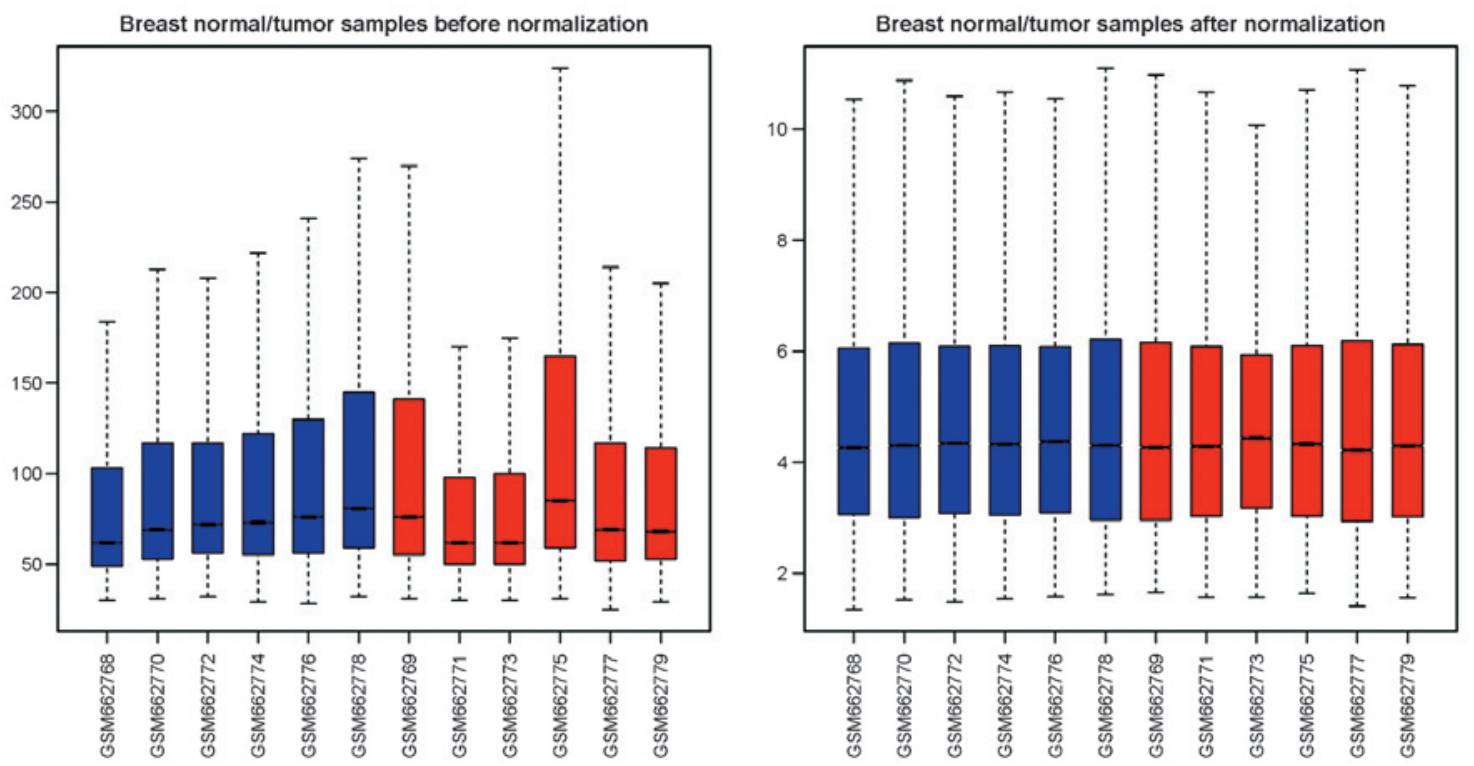

Figure 1. Box plots of results of data normalization. The $\mathrm{x}$ coordinate represents the samples and the $\mathrm{y}$-coordinate represents the gene expression values. The midline of the box plot represents the gene expression median and the whiskers represent the interquartile range. Blue box plots, normal breast tissue samples; red box plots, breast cancer tissue samples.

constructed. The present study aimed to generate a systematic perspective to understanding the underlying mechanisms and identifying novel therapeutic targets for breast cancer.

\section{Materials and methods}

Affymetrix microarray analysis. The array data for GSE26910, were downloaded from the Gene Expression Omnibus (GEO; http://www.ncbi.nlm.nih.gov/geo/) database, as reported by Planche et al (14). A total of 24 samples were used in the development of the Affymetrix microarray data. The expression profiles analyzed in this work were derived from 12 samples, including six samples of stroma surrounding invasive primary breast tumors and six samples of normal stroma breast tissues. The raw CEL data and annotation files were downloaded based on the GPL570 platform (Affymetrix Human Genome U133 Plus 2.0 Array; Affymetrix, Inc., Santa Clara, CA, USA) for further analysis.

Data processing and DEG analysis. The raw expression data were preprocessed using the robust multiarray average (15) algorithm with application of the Affy package (version 1.44.0; Fred Hutchinson Cancer Research Center, Seattle, WA, USA) in the R statistical software (version 3.1.2; Bell Labs, Murray Hill, NJ, USA). When multiple probes corresponded to the same gene, the mean value was calculated as the expression value of that gene.

The DEGs between breast cancer tissues and matched normal tissues were analyzed using the linear models for microarray data (limma) package (version 3.22.1; Fred Hutchinson Cancer Research Center) (16). Ilog of fold changel $>1$ and $\mathrm{P}<0.01$ were considered to be the cut-off values for DEG screening.

Gene ontology $(\mathrm{GO})$ and pathway enrichment analysis. GO is a tool for the unification of biology which collects structured, defined and controlled vocabulary for large scale of gene annotation (17). In addition, the Kyoto Encyclopedia of Genes and Genomes (KEGG; http://www.genome.jp/kegg/) database is used for the classification of correlating gene sets into their respective pathways (18).

In order to analyze the DEGs at a function level, GO annotation and KEGG pathway enrichment analyses for DEGs were performed using the Database for Annotation, Visualization and Integration Discovery (DAVID) software (version 6.7; http://david.abcc.ncifcrf.gov). The DEGs were classified into three GO categories, including molecular function (MF), biological process (BP) and cellular component (CC). $\mathrm{P}<0.01$ was set as the threshold value.

PPI network construction. Search Tool for the Retrieval of Interacting Genes (STRING), an online database resource that collects comprehensive information of predicted and experimental interactions of proteins (19), was used in the present study. The interactions of protein pairs in the STRING database were displayed using a combined score. The DEGs were mapped into PPI networks and a combined score of $>0.5$ was set as the cut-off value for significant protein pairs. The PPI network was established using Cytoscape software (version 1.1.1; National Institute of General Medical Sciences, Bethesda, MA, USA) (20) and the hub node was screened according to the degree score (number of neighbors). The subnetworks (nodes $>15$ ) were evaluated using the Molecular Complex Detection (MCODE) plugin of Cytoscape (21). Subsequently, the subnetwork functions were assessed by GO and pathway enrichment analyses of the genes involved in the subnetworks using the DAVID online tool.

\section{Results}

Data processing and DEG analysis. As shown in Fig. 1, the obscuring variations in the raw expression data were norma- 
Table I. GO and pathway enrichment analysis for DEGs.

\begin{tabular}{|c|c|c|c|}
\hline Category & Term & Count & P-value \\
\hline \multicolumn{4}{|l|}{ Upregulated DEGs } \\
\hline GOTERM_BP_FAT & GO: 0007155 - cell adhesion & 28 & $3.21 \times 10^{-7}$ \\
\hline GOTERM_BP_FAT & GO: 0022610 - biological adhesion & 28 & $3.30 \times 10^{-7}$ \\
\hline GOTERM_BP_FAT & GO: 0009611 - response to wounding & 22 & $4.93 \times 10^{-6}$ \\
\hline GOTERM_BP_FAT & GO: 0006955 - immune response & 21 & $6.08 \times 10^{-4}$ \\
\hline GOTERM_BP_FAT & GO: 0042981 - regulation of apoptosis & 21 & $3.74 \times 10^{-3}$ \\
\hline GOTERM_CC_FAT & GO: 0005576 - extracellular region & 68 & $3.07 \times 10^{-11}$ \\
\hline GOTERM_CC_FAT & GO: 0044421 - extracellular region part & 40 & $7.01 \times 10^{-9}$ \\
\hline GOTERM_CC_FAT & GO: 0005578 - proteinaceous extracellular matrix & 24 & $4.22 \times 10^{-10}$ \\
\hline GOTERM_CC_FAT & GO: 0031012 - extracellular matrix & 24 & $1.83 \times 10^{-9}$ \\
\hline GOTERM_CC_FAT & GO: 0005615 - extracellular space & 22 & $1.39 \times 10^{-3}$ \\
\hline GOTERM_MF_FAT & GO: 0005509 - calcium ion binding & 24 & $1.05 \times 10^{-3}$ \\
\hline GOTERM_MF_FAT & GO: 0030246 - carbohydrate binding & 14 & $4.94 \times 10^{-4}$ \\
\hline GOTERM_MF_FAT & GO: 0003779 - actin binding & 13 & $7.97 \times 10^{-4}$ \\
\hline GOTERM_MF_FAT & GO: 0042277 - peptide binding & 9 & $3.95 \times 10^{-3}$ \\
\hline GOTERM_MF_FAT & GO: 0005201 - extracellular matrix structural constituent & 8 & $9.73 \times 10^{-5}$ \\
\hline KEGG_PATHWAY & hsa04060: Cytokine-cytokine receptor interaction & 12 & $5.61 \times 10^{-3}$ \\
\hline KEGG_PATHWAY & hsa04510: Focal adhesion & 10 & $8.09 \times 10^{-3}$ \\
\hline KEGG_PATHWAY & hsa04512: Extracellular matrix-receptor interaction & 9 & $9.44 \times 10^{-5}$ \\
\hline \multicolumn{4}{|l|}{ Downregulated DEGs } \\
\hline GOTERM_BP_FAT & GO: 0007166 - cell surface receptor-linked signal transduction & 51 & $4.35 \times 10^{-4}$ \\
\hline GOTERM_BP_FAT & GO: 0009611 - response to wounding & 28 & $2.88 \times 10^{-7}$ \\
\hline GOTERM_BP_FAT & GO: 0007155 - cell adhesion & 27 & $1.29 \times 10^{-4}$ \\
\hline GOTERM_BP_FAT & GO: 0022610 - biological adhesion & 27 & $1.32 \times 10^{-4}$ \\
\hline GOTERM_BP_FAT & GO: 0010033 - response to organic substance & 26 & $4.93 \times 10^{-4}$ \\
\hline GOTERM_CC_FAT & GO: 0005886 - plasma membrane & 101 & $3.38 \times 10^{-6}$ \\
\hline GOTERM_CC_FAT & GO: 0005576 - extracellular region & 66 & $6.96 \times 10^{-7}$ \\
\hline GOTERM_CC_FAT & GO: 0044459 - plasma membrane part & 66 & $1.64 \times 10^{-5}$ \\
\hline GOTERM_CC_FAT & GO: 0044421 - extracellular region part & 46 & $1.76 \times 10^{-9}$ \\
\hline GOTERM_CC_FAT & GO: 0031226 - intrinsic to plasma membrane & 37 & $1.76 \times 10^{-3}$ \\
\hline GOTERM_MF_FAT & GO: 0030246 - carbohydrate binding & 18 & $1.02 \times 10^{-4}$ \\
\hline GOTERM_MF_FAT & GO: 0008289 - lipid binding & 17 & $3.87 \times 10^{-3}$ \\
\hline GOTERM_MF_FAT & GO: 0030247 - polysaccharide binding & 10 & $1.16 \times 10^{-3}$ \\
\hline GOTERM_MF_FAT & GO: 0001871 - pattern binding & 10 & $1.16 \times 10^{-3}$ \\
\hline GOTERM_MF_FAT & GO: 0005539 - glycosaminoglycan binding & 8 & $9.36 \times 10^{-3}$ \\
\hline KEGG_PATHWAY & hsa04360: Axon guidance & 8 & $9.98 \times 10^{-3}$ \\
\hline KEGG_PATHWAY & hsa04610: Complement and coagulation cascades & 7 & $1.69 \times 10^{-3}$ \\
\hline
\end{tabular}

Top five GO terms in various categories and significant pathways are listed. Count, number of DEGs enriched in a GO term/pathway; GO, gene ontology; DEGs, differentially expressed genes; MF, molecular function; BP, biological process; CC, cellular component; KEGG, Kyoto Encyclopedia of Genes and Genomes; FAT, function annotation test.

lized following preprocessing. Subsequently, DEG analysis was performed using the limma software package. A total of 571 genes were found to be differentially expressed between breast cancer tissues and normal tissues, among which 241 genes were upregulated and 330 genes were downregulated.

GO and pathway enrichment analyses. GO and pathway analyses were performed on upregulated and downregulated
DEGs, separately. The top five GO terms identified in each of the three $\mathrm{GO}$ categories (BP, CC and $\mathrm{MF}$ ) are shown in Table I. The overrepresented GO terms of upregulated DEGs were associated with cell adhesion, response to wounding, immune response, extracellular region, extracellular matrix, calcium ion binding and actin binding. The downregulated DEGs involved in the three GO categories were as follows: in the BP category, cell surface receptor-linked signal transduction, response to wounding and cell adhesion; in the CC 


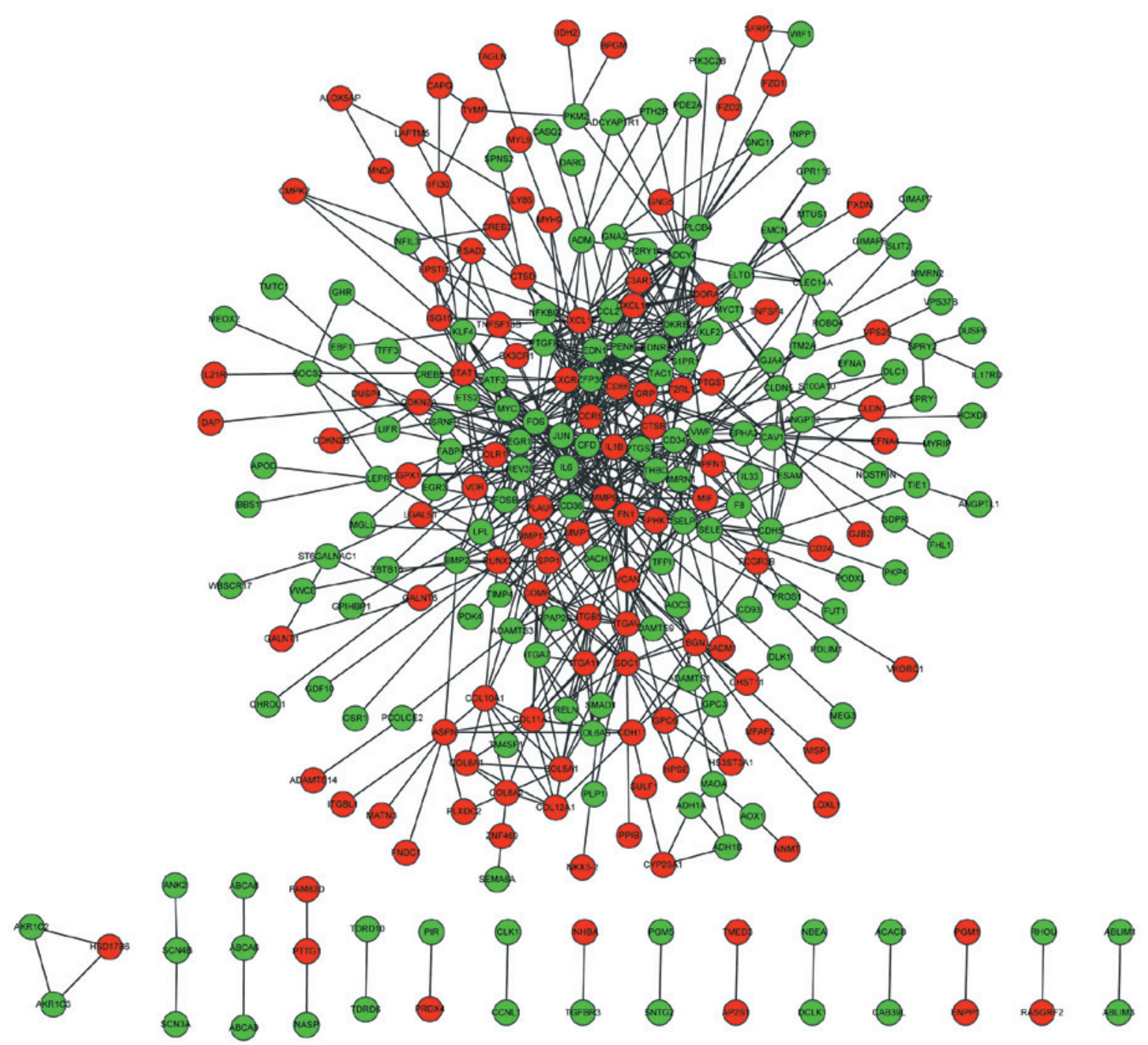

Figure 2. Protein-protein interaction network of differentially expressed genes. In the bottom panel, 20 single protein pairs with combined scores of 0.5 are shown, which presented no interaction with other proteins. Red nodes, upregulated genes; green nodes, downregulated genes.

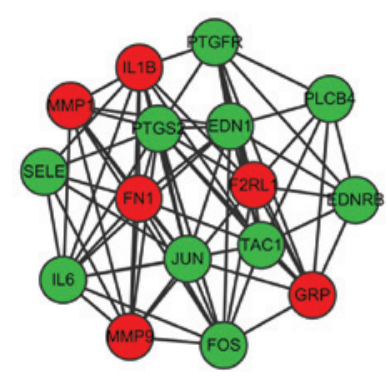

subnetwork1

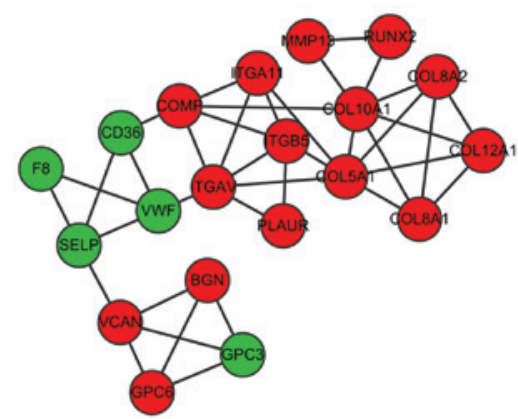

subnetwork2

Figure 3. Two subnetworks in the protein-protein interaction network. Red nodes, upregulated genes; green nodes, downregulated genes.

category, plasma membrane, extracellular region and plasma membrane region; and in the MF category, carbohydrate, lipid and polysaccharide binding.

The pathways significantly enriched by the upregulated DEGs included the cytokine-cytokine receptor interaction, focal adhesion and extracellular matrix (ECM)-receptor interaction pathways. By contrast, the two pathways that were enriched by the downregulated DEGs included the axon guidance and complement and coagulation cascade pathways (Table I).

PPI network analysis. Based on STRING database analysis, a total of 718 protein pairs with combined scores of $>0.5$ were identified. As demonstrated in Fig. 2, the PPI network consisted 
Table II. GO and pathway analysis for genes in subnetwork 1.

\begin{tabular}{llrl}
\hline Category & \multicolumn{1}{c}{ Term } & Count & P-value \\
\hline GOTERM_BP_FAT & GO: 0007166 - cell surface receptor linked signal transduction & 8 & $2.12 \times 10^{-3}$ \\
GOTERM_BP_FAT & GO: 0002237 - response to molecule of bacterial origin & 7 & $2.64 \times 10^{-10}$ \\
GOTERM_BP_FAT & GO: 0009617 - response to bacterium & 7 & $3.51 \times 10^{-8}$ \\
GOTERM_BP_FAT & GO: 0007610 - behavior & 7 & $6.45 \times 10^{-6}$ \\
GOTERM_BP_FAT & GO: 0009611 - response to wounding & 7 & $1.30 \times 10^{-5}$ \\
GOTERM_CC_FAT & GO: 0044421 - extracellular region part & 9 & $3.93 \times 10^{-6}$ \\
GOTERM_CC_FAT & GO: 0005576 - extracellular region & 9 & $8.38 \times 10^{-4}$ \\
GOTERM_CC_FAT & GO: 0005615 - extracellular space & $8.43 \times 10^{-6}$ \\
KEGG_PATHWAY & hsa05200: Pathways in cancer & 7 & $8.02 \times 10^{-5}$ \\
KEGG_PATHWAY & hsa04620: Toll-like receptor signaling pathway & 4 & $1.88 \times 10^{-3}$ \\
\hline
\end{tabular}

Top five GO terms in various categories and significant pathways are listed. Count, number of DEGs enriched in a GO term/pathway; GO, gene ontology; MF, molecular function; BP, biological process; CC, cellular component; KEGG, Kyoto Encyclopedia of Genes and Genomes; FAT, function annotation test.

Table III. GO and pathway analysis for genes in subnetwork 2.

\begin{tabular}{|c|c|c|c|}
\hline Category & Term & Count & P-value \\
\hline GOTERM_BP_FAT & GO: 0007155 - cell adhesion & 13 & $4.68 \times 10^{-12}$ \\
\hline GOTERM_BP_FAT & GO: 0022610 - biological adhesion & 13 & $4.76 \times 10^{-12}$ \\
\hline GOTERM_BP_FAT & GO: 0009611 - response to wounding & 7 & $4.36 \times 10^{-05}$ \\
\hline GOTERM_BP_FAT & GO: 0042060 - wound healing & 5 & $1.01 \times 10^{-04}$ \\
\hline GOTERM_BP_FAT & GO: 0001501 - skeletal system development & 5 & $7.15 \times 10^{-04}$ \\
\hline GOTERM_CC_FAT & GO: 0005576 - extracellular region & 15 & $2.88 \times 10^{-08}$ \\
\hline GOTERM_CC_FAT & GO: 0044421 - extracellular region part & 14 & $3.97 \times 10^{-11}$ \\
\hline GOTERM_CC_FAT & GO: 0005578 - proteinaceous extracellular matrix & 12 & $1.29 \times 10^{-13}$ \\
\hline GOTERM_CC_FAT & GO: 0031012 - extracellular matrix & 12 & $2.95 \times 10^{-13}$ \\
\hline GOTERM_CC_FAT & GO: 0009986 - cell surface & 8 & $3.97 \times 10^{-7}$ \\
\hline GOTERM_MF_FAT & GO: 0005509 - calcium ion binding & 7 & $7.77 \times 10^{-4}$ \\
\hline GOTERM_MF_FAT & GO: 0001948 - glycoprotein binding & 6 & $8.57 \times 10^{-10}$ \\
\hline GOTERM_MF_FAT & GO: 0005201 - extracellular matrix structural constituent & 5 & $4.00 \times 10^{-6}$ \\
\hline GOTERM_MF_FAT & GO: 0005539 - glycosaminoglycan binding & 5 & $2.76 \times 10^{-5}$ \\
\hline GOTERM_MF_FAT & GO: 0001871 - pattern binding & 5 & $4.02 \times 10^{-5}$ \\
\hline KEGG_PATHWAY & hsa04512: extracellular matrix-receptor interaction & 7 & $3.38 \times 10^{-9}$ \\
\hline KEGG_PATHWAY & hsa04510: Focal adhesion & 6 & $1.97 \times 10^{-5}$ \\
\hline KEGG_PATHWAY & hsa04610: Complement and coagulation cascades & 3 & $7.61 \times 10^{-3}$ \\
\hline KEGG_PATHWAY & hsa05412: Arrhythmogenic right ventricular cardiomyopathy & 3 & $9.18 \times 10^{-3}$ \\
\hline
\end{tabular}

Top five GO terms in various categories and significant pathways are listed. Count, number of DEGs enriched in a GO term/pathway; GO, gene ontology; MF, molecular function; BP, biological process; CC, cellular component; KEGG, Kyoto Encyclopedia of Genes and Genomes; FAT, function annotation test.

of 273 nodes and 718 edges. The nodes of fibronectin 1 (FN1; degree score, 39), interleukin 6 (IL6; degree score, 96) and c-Fos protein (degree score, 32 ) were hub proteins in the PPI network.

Two subnetworks (subnetworks 1 and 2) with $>15$ nodes were detected using the MCODE plugin (Fig. 3). The hub proteins FN1, IL6 and FOS were demonstrated to be involved in subnetwork 1 . Subnetwork 1 was mainly associated with response to wounding and extracellular region, while the most significant pathway was found to be the Toll-like receptor signaling pathway (Table II). By contrast, subnetwork 2 was associated with cell adhesion, response to wounding, wound healing, glycoprotein binding and calcium ion binding (Table III). In addition, the significant pathways associated with subnetwork 2 were focal adhesion, complement and coagulation cascades and arrhythmogenic right ventricular cardiomyopathy (Table III). 


\section{Discussion}

Breast cancer is the most common type of invasive cancer amongst females. In previous studies, gene expression profiling has been used to predict the clinical outcomes of breast cancer (22) and the distant metastasis of lymph-node-negative primary breast cancer (23). In the present study, using the gene expression patterns downloaded from the GEO database, 571 DEGs between breast cancer and normal tissues were identified, including 241 upregulated and 330 downregulated genes. GO analysis identified that several functional terms were statistically enriched by the DEGs, which were associated with cell adhesion, the extracellular region, response to wounding and immune response.

Cell adhesion is a common process in numerous biological processes, including cell-cell and cell-matrix interactions (24). Cell adhesion is mediated by multiple distinct families of receptors targeting cell adhesion to the ECM, as well as cellular growth, differentiation and migration (25). The tumor metastasis process initially requires the disruption of adhesion interaction between tumor and normal cells or the extracellular matrix, resulting in the release of neoplastic cells, followed by enhanced cell adhesion at later time-points (26). E-cadherin (E-cad) is a type of cell adhesion receptor, which modulates intercellular interactions in epithelial tissues (27). The critical role of E-cad in the invasion and metastasis of breast cancer cells has been previously reported (28). E-cad has been found to be significantly accumulated in breast cancer cells, accompanied by enhanced invasion and metastatic potential of tumor cells (28). Recent evidence has indicated that cellular adhesion molecules also possess prognostic significance in patients with breast cancer (29). Therefore, the expression of E-cad, combined with carcinoembryonic antigens, represents a powerful biomarker for predicting the prognosis of breast cancer.

In addition, cell adhesion is considered to be associated with the response to wounding and immune response. Cell migration of monolayers has been found to occur in response to wounding, surrounding the wound and facilitating wound closure (30). The activation of the immune response depends upon the regulation of cell-cell interactions in the immune system and cell adhesion receptor-regulation of the migration of lymphocytes and cell-cell interactions (30). The present study also demonstrated that the overrepresented pathways were associated with cell adhesion, including focal adhesion and ECM-receptor interactions. Therefore, cell adhesion may have a crucial role in mediating breast cancer development.

In order to explore the interactions of the identified DEGs, the PPI network was constructed. Three genes were identified to be significant nodes with maximum degrees, including $F N 1$, IL6 and FOS. In addition, these three genes were found to be significant nodes in subnetwork 1 (Fig. 3) and were involved in cell surface receptor-linked signal transduction, response to wounding and the extracellular region.

$F N 1$, also known as encoding fibronectin 1, is an ECM glycoprotein that binds to interleukin (31). FN1 was found to be involved in cell adhesion and migration, wound healing and host defense (32), which are in accordance with the functions of subnetwork 1 identified in the present study. The expression of FN1 is directly regulated by micro (mi)RNA-206, which has been demonstrated to be associated with metastatic cancer types, including breast cancer $(33,34)$. miRNA-206 inhibits cell growth in breast cancer by targeting estrogen receptor 1. Furthermore, FN1 was found to be correlated with the drug-resistance of cancer cells (35). The expression of $F N 1$ was reported to be significantly accumulated in vincristine-resistant myeloma cells, while other ECM components, including type II collagen $\alpha 1$, were downregulated. The results of the present study indicated that the FN1 gene was upregulated in the development of breast cancer and that FN1 was a hub protein with a degree score of 39 in the established PPI network. Therefore, the $F N 1$ gene was found to be a key regulator in breast cancer development.

FOS is a family of transcription factors including c-Fos, FosB and Fra-1 (36). c-Fos is a proto-oncogene associated with cellular functions and has been found to be overexpressed in various types of cancer. $c$-Fos functions as a nuclear transcription factor and plays a crucial role in growth factor signaling (37). $c$-Fos is one of the targets for the estrogen receptor (38), and the expression of $c$-Fos is significantly enhanced by the induction of estrogen in breast cancer cells (39). Estrogen sensitizes breast cancer cells to growth factors, which then contribute to tumor growth (37). In addition, previous studies have indicated that proto-oncogenes, including $c$-Fos, are involved in breast cancer cell cycle-associated functions (40). Progestins, as members of the estrogen family, have been shown to enhance $c$-Fos expression resulting in acceleration of the cell cycle progression (41). Furthermore, IL6 is recognized as a pro-inflammatory cytokine that modulates the inflammatory response (42). The role of IL6 signaling has been widely investigated in the development of various types of cancer, including liver (43), lung (44) and breast cancer (45). A recent study reported that the expression levels of $I L 6$ and $I L 8$ in triple-negative breast cancer (TNBC) were associated with cell survival, and that the inhibition of IL6/IL 8 signaling was a therapeutic strategy for improving the prognosis of patients with TNBC (45). Therefore, the key role of FN1, IL6 and FOS in breast cancer development has been demonstrated. These nodes may provide promising targets for the treatment of breast cancer in the future.

In conclusion, gene expression profiles were found to be altered during the development and progression of breast cancer. The cell adhesion, extracellular region and immune response were significant functions of the DEGs identified in breast cancer progression. In addition, the FNI, IL6 and FOS genes were found to be involved in breast cancer development. The present study suggested that FN1, IL6 and FOS may be potential targets in the development of treatments for breast cancer. However, further evaluation of their potential applications is required.

\section{References}

1. Powles TJ: Breast cancer prevention. Oncologist 7: 60-64, 2002.

2. Yager JD and Davidson NE: Estrogen carcinogenesis in breast cancer. N Engl J Med 354: 270-282, 2006.

3. Nathanson KN, Wooster R and Weber BL: Breast cancer genetics: what we know and what we need. Nat Med 7: 552-556, 2001.

4. Wemer RS, McCormick B, Petrec J, et al: Arm edema in conservatively managed breast cancer: obesity is a major predictive factor. Radiology 180: 177-184, 1991.

5. Clapp RW, Jacobs MM and Loechler EL: Environmental and occupational causes of cancer: new evidence 2005-2007. Rev Environ Health 23: 1-37, 2008. 
6. Mohankumar K, Pajaniradje S, Sridharan S, et al: Mechanism of apoptotic induction in human breast cancer cell, MCF-7, by an analog of curcumin in comparison with curcumin - an in vitro and in silico approach. Chem Biol Interact 210: 51-63, 2014.

7. World Health Organization: World cancer report 2008. Boyle P and Levin B (eds). IARC 2008.

8. Price JT, Tiganis T, Agarwal A, Djakiew D and Thompson EW: Epidermal growth factor promotes MDA-MB-231 breast cancer cell migration through a phosphatidylinositol 3'-kinase and phospholipase C-dependent mechanism. Cancer Res 59 5475-5478, 1999.

9. Dang TT and Pearson GW: Abstract A38: Breast cancer intrinsic subtype specific interactions with the microenvironment dictate the mechanism of tumor invasion. Cancer Res 73 (3 Supplement): A38-A38, 2013.

10. Wu K, Weng Z, Tao Q, et al: Stage-specific expression of breast cancer-specific gene $\gamma$-synuclein. Cancer Epidemiol Biomarkers Prev 12: 920-925, 2003

11. Shou J, Massarweh S, Osborne CK, et al: Mechanisms of tamoxifen resistance: increased estrogen receptor-HER2/neu cross-talk in ER/HER2-positive breast cancer. J Natl Cancer Inst 96: 926-935, 2004.

12. Pietras RJ, Arboleda J, Reese DM, et al: HER-2 tyrosine kinase pathway targets estrogen receptor and promotes hormone-independent growth in human breast cancer cells. Oncogene 10 2435-2446, 1995.

13. Kubo M, Nakamura M Tasaki A, et al: Hedgehog signaling pathway is a new therapeutic target for patients with breast cancer. Cancer Res 64: 6071-6074, 2004.

14. Planche A, Bacac M, Provero P, et al: Identification of prognostic molecular features in the reactive stroma of human breast and prostate cancer. PLoS One 6: e18640, 2011.

15. Irizarry RA, Hobbs B, Collin F, et al: Exploration, normalization, and summaries of high density oligonucleotide array probe level data. Biostatistics 4: 249-264, 2003.

16. Smyth GK: Linear models and empirical bayes methods for assessing differential expression in microarray experiments. Stat Appl Genet Mol Biol 3: Article3, 2004.

17. Ashburner M, Ball CA, Blake JA, et al: Gene ontology: tool for the unification of biology. Nat Genet 25: 25-29, 2000.

18. Altermann E and Klaenhammer TR: PathwayVoyager: pathway mapping using the kyoto encyclopedia of genes and genomes (KEGG) database. BMC Genomics 6: 60, 2005.

19. Szklarczyk D, Franceschini A, Kuhn M, et al: The STRING database in 2011: functional interaction networks of proteins, globally integrated and scored. Nucleic Acids Res 39: D561-D568, 2011.

20. Kohl M, Wiese S and Warscheid B: Cytoscape: software for visualization and analysis of biological networks. Methods Mol Biol 696: 291-303, 2011.

21. Bader GD and Hogue CW: An automated method for finding molecular complexes in large protein interaction networks. BMC Bioinformatics 4: 2, 2003.

22. van 't Veer LJ, Dai H, van de Vijver MJ, et al: Gene expression profiling predicts clinical outcome of breast cancer. Nature 415 : 530-536, 2002

23. Wang Y, Klijn JG, Zhang Y, et al: Gene-expression profiles to predict distant metastasis of lymph-node-negative primary breast cancer. Lancet 365: 671-679, 2005.

24. Gumbiner BM: Cell adhesion: The molecular basis of tissue architecture and morphogenesis. Cell 84: 345-357, 1996.

25. Albelda SM and Buck CA: Integrins and other cell adhesion molecules. FASEB J 4: 2868-2880, 1990.

26. Albelda S: Role of integrins and other cell adhesion molecules in tumor progression and metastasis. Lab Invest 68: 4-17, 1993.
27. Siitonen SM, Kononen JT, Helin HJ, Rantala IS, Holli KA and Isola JJ: Reduced E-cadherin expression is associated with invasiveness and unfavorable prognosis in breast cancer. Am J Clin Pathol 105: 394-402, 1996.

28. Oka H, Shiozaki H, Kobayashi K, et al: Expression of E-cadherin cell adhesion molecules in human breast cancer tissues and its relationship to metastasis. Cancer Res 53: 1696-1701, 1993.

29. Saadatmand S, De Kruijf E, Sajet A, et al: Expression of cell adhesion molecules and prognosis in breast cancer. Br J Surg 100: 252-260, 2013.

30. Springer TA: Adhesion receptors of the immune system. Nature 346: 425-434, 1990.

31. Pankov R and Yamada KM: Fibronectin at a glance. J Cell Sci 115: 3861-3863, 2002.

32. Steffens S, Schrader A, Vetter G, et al: Fibronectin 1 protein expression in clear cell renal cell carcinoma. Oncol Lett 3: 787-790, 2012.

33. Kondo N, Toyama T, Sugiura H,Fujii Y and Yamashita H: miR-206 expression is down-regulated in estrogen receptor alpha-positive human breast cancer. Cancer Res 68: 5004-5008, 2008.

34. Adams BD, Claffey KP and White BA: Argonaute-2 expression is regulated by epidermal growth factor receptor and mitogen-activated protein kinase signaling and correlates with a transformed phenotype in breast cancer cells. Endocrinology 150 14-23, 2009.

35. Mutlu P, Ural AU and Gunduz U: Differential gene expression analysis related to extracellular matrix components in drug-resistant RPMI-8226 cell line. Biomed Pharmacother 66: 228-231, 2012

36. Milde-Langosch K: The Fos family of transcription factors and their role in tumourigenesis. Eur J Cancer 41: 2449-2461, 2005.

37. Lee AV, Cui X and Oesterreich S: Cross-talk among estrogen receptor, epidermal growth factor, and insulin-like growth factor signaling in breast cancer. Clin Cancer Res 7: 4429s-4435s, 2001.

38. Hanstein B, Djahansouzi S, Dall P, Beckmann M and Bender H: Insights into the molecular biology of the estrogen receptor define novel therapeutic targets for breast cancer. Eur J Endocrinol 150: 243-255, 2004.

39. Morishita S, Niwa K, Ichigo S, et al: Overexpressions of c-fos/jun mRNA and their oncoproteins (Fos/Jun) in the mouse uterus treated with three natural estrogens. Cancer Lett 97: 225-231, 1995.

40. Musgrove EA, Lee C and Sutherland RL: Progestins both stimulate and inhibit breast cancer cell cycle progression while increasing expression of transforming growth factor alpha, epidermal growth factor receptor, c-fos, and c-myc genes. Mol Cell Biol 11: 5032-5043, 1991.

41. Sutherland RL, Prall OW, Watts CK and Musgrove EA: Estrogen and progestin regulation of cell cycle progression. J Mammary Gland Biol Neoplasia 3: 63-72, 1998.

42. Xing Z, Gauldie J, Cox G, Baumann H, Jordana M, Lei XF and Achong MK: IL-6 is an antiinflammatory cytokine required for controlling local or systemic acute inflammatory responses. J Clin Invest 101: 311-320, 1998.

43. He G, Dhar D, Nakagawa H, et al: Identification of liver cancer progenitors whose malignant progression depends on autocrine IL-6 signaling. Cell 155: 384-396, 2013.

44. Seifart C, Plagens A, Dempfle A, et al: TNF- $\alpha$, TNF- $\beta$, IL-6, and IL-10 polymorphisms in patients with lung cancer. Dis Markers 21: 157-165, 2013.

45. Hartman ZC, Poage GM, Den Hollander P, et al: Growth of triple-negative breast cancer cells relies upon coordinate autocrine expression of the proinflammatory cytokines IL- 6 and IL-8. Cancer Res 73: 3470-3480, 2013. 